\section{Lamp for tadpoles}

To the editor: We would like to draw the attention of your readers to two recent articles that we believe will be important for the detection of parasite infections, particularly in the field setting. The first of these two procedures uses loop-mediated isothermal amplification (LAMP) and the second uses protein-DNA chimeras.

The first procedure, LAMP, is a recently introduced alternative to PCR (ref. 1 and Eiken Chemical Company (Japan) website and references therein (http://loopamp.eiken.co.jp/)). LAMP is a technology based on Bst DNA polymerase and, compared to standard PCR, has advantages of reaction simplicity, specificity and detection sensitivity. Furthermore, because LAMP does not require complicated thermal cycling steps (an isothermal reaction at $63-65^{\circ} \mathrm{C}$ is sufficient), heating for about $1 \mathrm{~h}$ in a simple incubator such as a water bath or a block heater is sufficient to amplify the target DNA to detectable levels $(>10 \mu \mathrm{g}$ of target DNA within 30-60 min). A useful by-product of the reaction is the copious white precipitate of magnesium pyrophosphate, which allows easy and rapid visual confirmation that the target DNA was amplified by LAMP. Notably, Bst DNA polymerase used in LAMP is not affected by blood-derived tissue components such as myoglobin, heme-blood protein complexes and immunoglobulin G that can inactivate Taq DNA polymerase used in PCR. We have proposed that LAMP would be a highly sensitive method for the detection of African trypanosomes - particularly in the field, and in local clinics and hospitals where cost and environmental constraints prohibit the use of PCR ${ }^{2}$. LAMP has been shown to be a simple, rapid and cost-effective method for monitoring environmental bacteria and for the detection and/or clinical diagnosis of Coxiella burnetii, Listeria monocytogenes, SARS coronavirus, Yersinia pseudotuberculosis and Mycobacterium avium.

The second procedure, described by Ian Burbulis and colleagues, makes use of 'inteins' to covalently tag a 'DNA barcode' to a single site on a protein to create a protein-DNA hybrid called a 'tadpole ${ }^{3-5}$. The protein portion of the tadpole serves as recognition motif for the target compound, and the DNA barcode contains a region for PCR amplification as well as a T7 RNA polymerase binding and a transcription start site for RNA-mediated detection. By amplifying the tadpoles' DNA by PCR they obtained results with great dynamic range and specificity. For example, the detection of 'protective antigen' subunit of the Bacillus anthracis toxin in serum was 200 times more sensitive by the tadpole procedure than the corresponding ELISA assay. Such a range of ultrasensitive analyses should have an important potential application for pathogen detection, disease diagnosis and environmental monitoring ${ }^{3-5}$.

Alone, the above two procedures are clearly very powerful. But if the procedures were to be combined so that the Bst DNA polymerase procedure of LAMP replaced the PCR of the tadpole procedure, we believe an even more powerful technique would be developed. The combined technology should be easier and potentially more specific, and it should provide a 'visual' check that the procedure is functioning normally. This visual procedure could be used instead of one that requires sophisticated equipment, such as microtiter plate readers, which do not always work well in a third-world setting. The combination of technologies could be useful also in developed countries. Ultrasensitive visual diagnostic tests could be easily conducted in a laboratory or a physician's office. When quantitative analysis is important, quantitative realtime LAMP is also available. Essentially any use that can be found for the tadpole technology using PCR readout will be enhanced by the advantages of LAMP.

\section{Dennis J Grab ${ }^{1}$, J ohn Lonsdale-Eccles ${ }^{2}$ \& Noboru Inoue ${ }^{3}$}

${ }^{1}$ Department of Pediatrics, Johns Hopkins University School of Medicine, 600 North Wolfe Street, Park 256, Baltimore, Maryland 21287, USA. ${ }^{2} 4720$ Grant Drive, Gadsden, Alabama 35907, USA. ${ }^{3}$ National Research Center for Protozoan Diseases, Obihiro University of Agriculture and Veterinary Medicine, 2-13 Inada, Obihiro, Hokkaido 080-8555, Japan.

e-mail:dgrab@jhmi.edu

1. Notomi, T. et al. Nucleic Acids Res. 28, e63 (2000).

2. Kuboki, N. et al. J. Clin. Microbiol. 41, 5517-5524 (2003).

3. Burbulis, I., Yamaguchi, K., Gordon, A., Carlson, R. \& Brent, R. Nat. Methods 2, 31-37 (2005).

4. Nolan, G.P. Nat. Methods 2, 11-12 (2005)

5. Evans, J. Chemistry World 2, 18 (2005).

Brent and Burbulis reply: We find the comments of Grab, LonsdaleEccles and Inoue to be well taken.

The presence of a DNA tail in tadpole chimeric molecules allows them to be used with any means to detect, amplify and quantify DNA. Our first methods that used these molecules used linear amplification with T7 RNA polymerase ${ }^{1}$ and exponential amplification by the TaqMan real-time PCR method ${ }^{2}$. We chose this real-time PCR amplification method because it has a large installed user base, and because we were confident we would be able to revisit the statistics used to quantify its output to increase the sensitivity and accuracy of measurements made using it.

Although we do not greatly favor (or disfavor) any particular nucleic acid amplification, detection and quantification methods, we agree that LAMP and other 'isothermal' methods with simple readouts offer significant notional advantages, particularly for applications in the developing world where access to capital equipment, reliable electrical power and reliable refrigeration for reagents may be difficult. We also believe that although the statistics and calibration methods will be different for each method, similar careful attention to the statistics used to quantify 
the output will be able to produce measurements of great sensitivity and precision.

Finally, we note that at present, application of sensitive molecular detection methods in the developing world, as well as in the developed world, including detection of common infectious disease and routine health assessment via serum proteomics, will be made difficult by the cost and difficulty of aggregating licenses to existing patents covering the different steps of the methods. It may also be hampered by a need to operate through an existing industrial to perceived higher-return applications such as diagnosis of cancer and chronic diseases of affluent countries. Widespread application of sensitive molecular detection methods to other applications may require modification of licensing frameworks to facilitate aggregation of intellectual property to combine the experimental steps. It may also require deliberate attention to the creation of a modified industrial structure that allows profitable (or subsidized) deployment of these methods at lower price points.

\section{Roger Brent \& Ian Burbulis}

The Molecular Sciences Institute, 2168 Shattuck Avenue, Berkeley, California 94704, USA.

e-mail: brent@molsci.org or burbulis@molsci.org

1. Zhang, H.T., Kacharmina, J.E., Miyashiro, K. Greene, M.I. \& Eberwine, J. Proc. Natl. Acad. Sci. USA 98, 5497-5502 (2001).

2. Higuchi, R., Fockler, C., Dollinger, G. \& Watson, R. Biotechnology 11, 10261030 (1993). 